\title{
Analysis of the Interference Modulation Depth in the Fourier Transform Spectrometer
}

\author{
Rilong Liu \\ School of Engineering, Jiangxi Agricultural University, Nanchang 330045, China \\ Correspondence should be addressed to Rilong Liu; ckjx312@aliyun.com
}

Received 21 August 2015; Revised 14 September 2015; Accepted 20 September 2015

Academic Editor: Michele Norgia

Copyright (C) 2015 Rilong Liu. This is an open access article distributed under the Creative Commons Attribution License, which permits unrestricted use, distribution, and reproduction in any medium, provided the original work is properly cited.

Based on the principle of the Michelson interferometer, the paper briefly describes the theoretical significance and calculates and deduces three expressions of the interference modulation depth. The influence of the surface shape error of plane mirror on modulation depth is analyzed, and the tolerance of error is also pointed out. Moreover, the dependence of modulation depth on the reflectance change of beam splitter interface is also analyzed, and the curve is given. It is concluded that this paper is of general significance for the Fourier transform spectrometer based on the principle of the Michelson two-beam interference.

\section{Introduction}

The Fourier transform spectrometer is an instrument which is used to analyze the spectral distribution of the light source by means of the interference effect based on the Fourier transform technology. It has incomparably more advantages than conventional spectral instruments in acquiring higher spectral resolution. There are many kinds of Fourier transform spectrometers, including Michelson interferometer, Fabry-Perot interferometer, Sagnac interferometer, and double refraction interferometer $[1,2]$.

The classical Michelson interferometer consists of a moving mirror and a fixed mirror. The two beams of lights, reflected by these two mirrors, make up the phase difference. When the moving mirror moves, the intensity of interference signals is modulated to form the interferogram. The modulation depth is also known as the interference modulation efficiency. It is one of the most important factors influencing the signal-to-noise ratio of the interferogram, and a change in it will significantly affect the sensitivity of the spectrometer [3, 4]. Based on the Michelson type Fourier transform spectrometer, this paper explores the optical principle of interference system through a quantitative analysis of influencing factors of surface shape of mirror and performance change of beam splitter. It is hoped that suggestions can be made for the obtaining of ideal interferogram.

\section{The Modulation Depth of Fourier Transform Spectrometer}

The most classical Fourier transform spectrometer is the Michelson interferometer, which is composed of a fixed mirror, a moving mirror, a beam splitter, and a detector. Its basic structure is shown in Figure 1.

In the Michelson interferometer, the fixed mirror and the moving mirror are perpendicular to each other, with an angle of $45^{\circ}$ between the beam splitter and two mirrors. The collimated beam shines the interference system, and the beam splitter separates the incident light into two beams with roughly the same reflectance and transmittance. One reflected beam by the fixed mirror then goes through the beam splitter again, whereas the other transmitted beam is reflected by the moving mirror and divided by the beam splitter. Because these two beams of light are produced by the same beam, they have a constant phase difference. When these two beams of light travel through a convergent lens, the interference is formed on the detector.

Upon arrival at the detector, the two coherent beams have an optical path difference for the shift of the moving mirror. The optical path difference then shows periodic changes with round-trip translation of the moving mirror. According to the principle of interference spectroscopy, the interference signals can be obtained on the detector. 


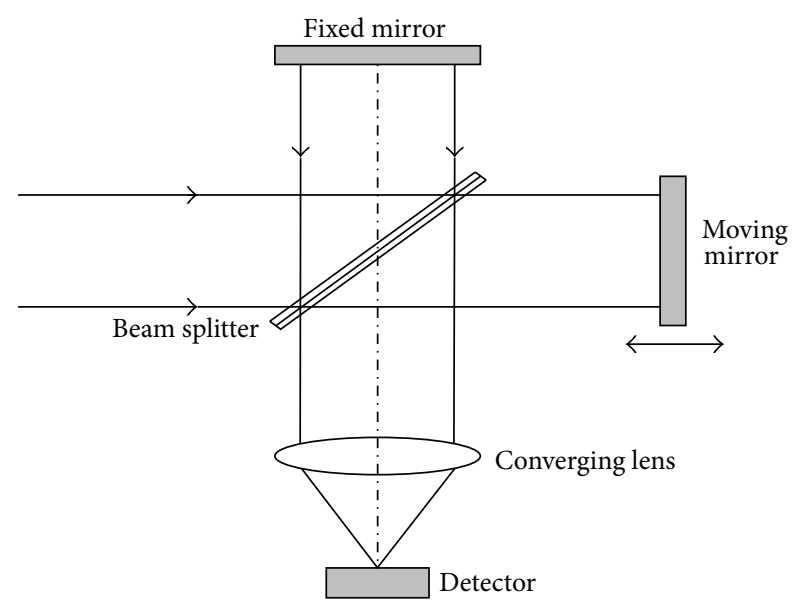

FIGURE 1: Basic structure of Michelson interferometer.

According to the interference theory, the intensity of interference signals generated by the two-beam interference is [5]

$$
\begin{aligned}
I & =I_{1}+I_{2}+2 \sqrt{I_{1} I_{2}} \cos \delta \\
& =\left(I_{1}+I_{2}\right)\left(1+\frac{2 \sqrt{I_{1} I_{2}}}{I_{1}+I_{2}} \cos \delta\right),
\end{aligned}
$$

where $I_{1}$ and $I_{2}$ are, respectively, the intensity of two coherent beams and $\delta$ is the phase difference of two coherent beams.

The definition of the modulation depth of interference signals, namely, the visibility of interference signals $M$, is

$$
M=\frac{I_{\max }-I_{\min }}{I_{\max }+I_{\min }} .
$$

This equation indicates the degree of bright dark contrast of fringes in the interference field, where $I_{\max }$ and $I_{\min }$ stand for the maximum intensity and the minimum intensity of interference signals. We then get the following equation:

$$
M=\frac{I_{\max }-I_{\min }}{I_{\max }+I_{\min }}=\frac{2 \sqrt{I_{1} I_{2}}}{I_{1}+I_{2}} .
$$

Then (1) can be rewritten as

$$
I=\left(I_{1}+I_{2}\right)(1+M \cos \delta) .
$$

From (3), we can have the coefficient of the cosine, namely, the modulation depth, after we obtain the distribution of the cosine light intensity, and normalize the constant term. If and only if $I_{1}=I_{2}=I_{0}$, that is to say, when the intensity of two coherent beams of light is equal, the modulation depth reaches a maximum and also an ideal $M=1$. However, with practical applications taken into consideration, the modulation depth $M$ will usually be less than 1 .

\section{The Surface Shape Error of Plane Mirror}

The fixed mirror and the moving mirror of Fourier transform spectrometer are composed of plane mirrors with a rectangular aperture. In the process of making plane mirrors, there are a number of factors modulating the surface error, such as polishing, coating, and mirror bending. It follows that these mirrors are not ideal surface shape, and there is always a certain surface error. Therefore, in a spectrometer composed of these mirrors, the modulation depth is often not ideal. In what follows, we will analyze the influence of curve error and polishing error of plane mirror on interference modulation depth. Suppose polishing error of mirror obeys normal distribution with zero mean, and its maximum fluctuation is $\varepsilon_{\text {max }}$. In addition, curve error of mirror is subject to uniform distribution, and its maximum curvature is $\varsigma_{\max }$. Assume also that other optical components in Fourier transform spectrometer are ideal.

When there is a surface shape error of plane mirror, the reflection of two beams of coherent light will generate wavefront distortion, which results in a change in optical path difference of interferometer. The phase difference $\delta$ of the two beams of coherent light changes accordingly. For a small change $\xi$ of the surface shape error, the change of the phase difference is

$$
\Delta \delta=2 \pi \nu \mathcal{E}
$$

where $v$ is the wave number of the incident light. Suppose $f(\varepsilon)$ is the probability density function of surface shape error. If $\varepsilon \in(\varepsilon, \Delta \varepsilon)$, then we obtain surface $d S=f(\varepsilon) d \varepsilon$. Because of the effect of surface shape error, the intensity of interference signals is [6]

$$
I=\frac{\int_{S} I^{\prime}(\delta) d S}{\int_{S} d S}
$$

where $I^{\prime}(\delta)$ represents the distribution of interference intensity in any one point. The probability density function is

$$
f(\varepsilon)=\frac{1}{\sqrt{2 \pi} \sigma} \exp \left\{-\frac{\varepsilon^{2}}{2 \sigma^{2}}\right\},
$$

where $\sigma$ is the standard deviation of $\varepsilon$.

When $d S=f(\varepsilon) d \varepsilon$ is substituted to (6), we can get the intensity of interference signals:

$I$

$$
\begin{aligned}
& =\frac{\int_{-\infty}^{+\infty} 2 I_{0}(1+\cos (\delta+2 \pi \nu \varepsilon))(1 / \sqrt{2 \pi} \sigma) \exp \left(-\varepsilon^{2} / 2 \sigma^{2}\right) d \varepsilon}{\int_{-\infty}^{+\infty}(1 / \sqrt{2 \pi} \sigma) \exp \left(-\varepsilon^{2} / 2 \sigma^{2}\right) d \varepsilon} \\
& =2 I_{0}\left[1+\exp \left(-\frac{(2 \pi \nu \sigma)^{2}}{2}\right) \cos \delta\right] .
\end{aligned}
$$

Hence, the interference modulation depth is

$$
M_{1}=\exp \left\{-\frac{(2 \pi \nu \sigma)^{2}}{2}\right\} \text {. }
$$


It can be seen that the interference modulation depth is inversely proportional to the variance of polishing error of mirror, which means that a good polishing surface can improve the modulation depth. If the interference modulation depth is set at $M_{1} \geq 0.9$, the error tolerance of standard deviation $\sigma$ is

$$
\sigma \leq \frac{\lambda}{14} .
$$

In the same way, when the mirror surface is curved, the existence of the curve error causes the intensity distribution of the interference signals to be

$$
\begin{aligned}
& I \\
& =\frac{\int_{-\varsigma_{\max }}^{+\zeta_{\max }} 2 I_{0}(1+\cos (\delta+2 \pi \nu \varsigma))\left(1 /\left(\varsigma_{\max }-\left(-\varsigma_{\max }\right)\right)\right) d \varsigma}{\int_{-\varsigma_{\max }}^{+\zeta_{\max }}\left(1 /\left(\varsigma_{\max }-\left(-\varsigma_{\max }\right)\right)\right) d \varsigma} \\
& =2 I_{0}\left[1+\frac{\sin \left(2 \pi \nu \varsigma_{\max }\right)}{2 \pi \nu \varsigma_{\max }} \cos \delta\right] .
\end{aligned}
$$

It follows that, when the surface of mirror is deformed, the interference modulation depth is

$$
M_{2}=\frac{\sin \left(2 \pi \nu \varsigma_{\max }\right)}{2 \pi \nu \varsigma_{\max }} .
$$

Equation (12) shows that $M_{2}$ is a function of sinc, and the modulation depth $M_{2}$ decreases when the curvature $\varsigma$ increases. If the interference modulation depth is set at $M_{2} \geq$ 0.9 , the error tolerance of the curvature $\varsigma$ is

$$
\varsigma \leq \frac{\lambda}{8} \text {. }
$$

Through the above analysis, we know that the surface error of mirror has an effect on the modulation depth. In order to achieve better interference effect, the surface error of mirror should meet error tolerance requirements.

\section{The Reflectance Change of Beam Splitter Interface}

In the Michelson interferometer shown in Figure 1, the separating surface of the beam splitter is coated with transflective film. Suppose $r_{i}$ and $t_{i}$ represent the amplitude reflection coefficient and the amplitude transmission coefficient of beam from the surrounding medium into surface $i$ of the beam splitter, respectively. Meanwhile, $r_{i}^{\prime}$ and $t_{i}^{\prime}$ are the amplitude reflection coefficient and the amplitude transmission coefficient of beam from surface $i$ of the beam splitter into the surrounding medium, respectively. At the same time, $r_{f}$ is the amplitude reflection coefficient of fixed mirror, and $r_{m}$ is the amplitude reflection coefficient of moving mirror, and $A$ stands for the amplitude of incident light $[7,8]$. The complex amplitude of the two beams of light reflected by Michelson interferometer, respectively, is

$$
\begin{aligned}
& E_{1}=t_{1} r_{2}^{\prime} t_{1}^{\prime} r_{f} t_{1} t_{2}^{\prime} A \exp \left(j 2 \pi v \chi_{1}\right), \\
& E_{2}=t_{1} t_{2}^{\prime} r_{m} r_{2} A \exp \left(j 2 \pi v \chi_{2}\right),
\end{aligned}
$$

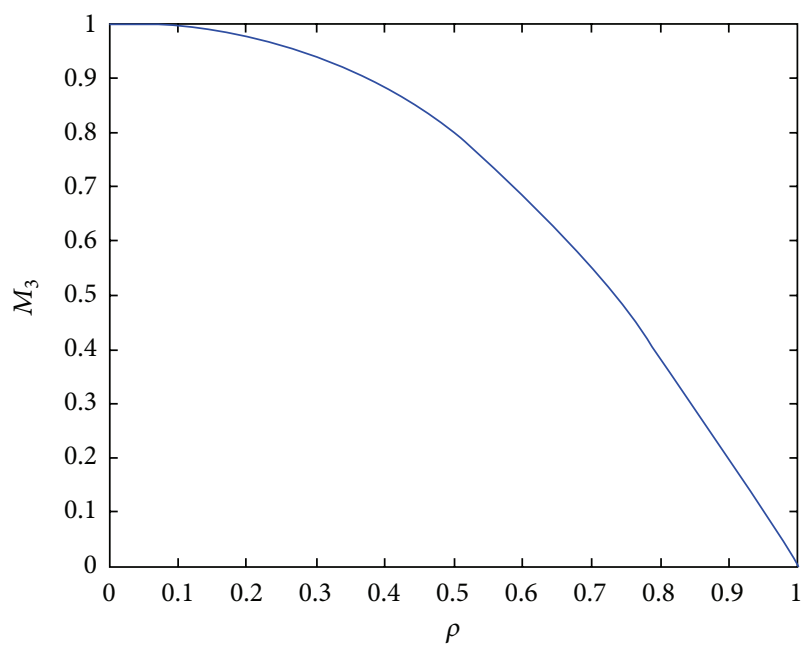

FIGURE 2: Influence of reflectance change of beam splitter on modulation depth.

where $v$ is the wave number of the incident light, while $\chi_{1}$ and $\chi_{2}$ represent optical path of two beams of light, respectively. Generally speaking, if the amplitude reflection coefficient of the fixed mirror equals that of the moving mirror, that is, $r_{f}=$ $r_{m}$, then we get

$$
\frac{E_{1}}{E_{2}}=\frac{r_{2}^{\prime} t_{1} t_{1}^{\prime}}{r_{2}} \exp \left(i 2 \pi v\left(\chi_{1}-\chi_{2}\right)\right) .
$$

From (15), the modulation depth can be expressed as

$$
M_{3}=\frac{2\left|E_{1} / E_{2}\right|}{1+\left|E_{1} / E_{2}\right|^{2}}=\frac{2\left|\left(r_{2}^{\prime} t_{1} t_{1}^{\prime}\right) /\left(r_{2}\right)\right|}{1+\left|\left(r_{2}^{\prime} t_{1} t_{1}^{\prime}\right) /\left(r_{2}\right)\right|^{2}} .
$$

According to the Fresnel formula, the amplitude reflection coefficient and the amplitude transmission coefficient satisfy the following conditions:

$$
\begin{gathered}
r_{2}=-r_{2}^{\prime}, \\
t_{1} t_{1}^{\prime}=1-r_{1}^{2} .
\end{gathered}
$$

And the interface reflectance of the beam splitter is

$$
\rho=r_{1}^{2} .
$$

Then substituting (18) into (17), we can get

$$
t_{1} t_{1}^{\prime}=1-\rho .
$$

Thus, the interference modulation depth is

$$
M_{3}=\frac{2-2 \rho}{2-2 \rho+\rho^{2}} .
$$

From (20), it can be seen that the interference modulation depth $M_{3}$ will change with the interface reflectance $\rho$ of the beam splitter. Their relationship is captured in Figure 2.

From Figure 2, the smaller the interface reflectance $\rho$ of the beam splitter is, the greater the interference modulation 


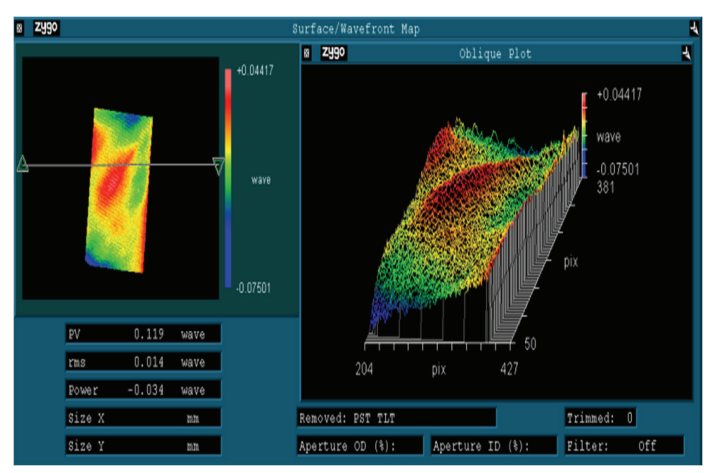

FIGURE 3: The surface shape of plane mirror.

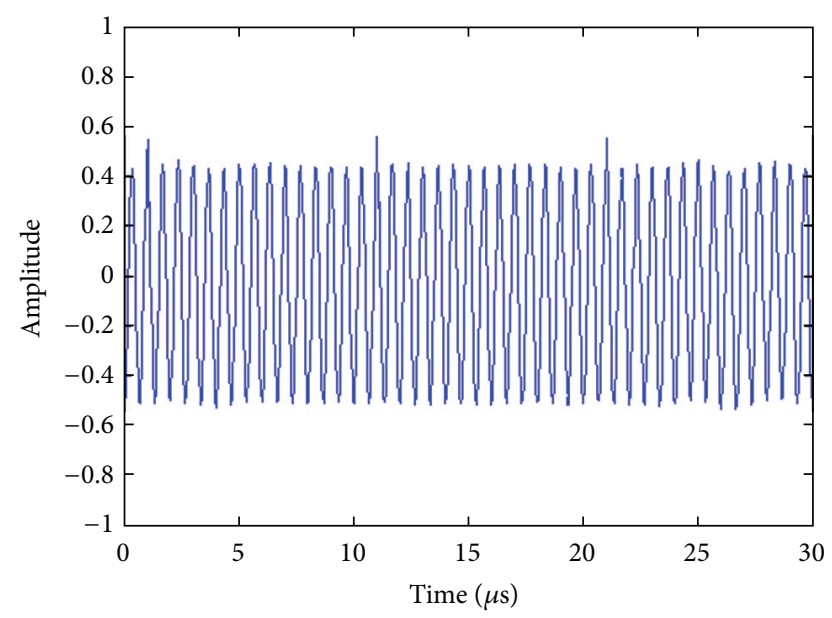

FIGURE 4: The electrical signal of the laser interference.

depth $M_{3}$ becomes. To put it another way, if $\rho$ value is 0 , then $M_{3}=1$. When the interference modulation depth is set at $M_{3} \geq 0.9, \rho$ is

$$
\rho \leq 0.372
$$

\section{Experimental Results}

The analysis of interference modulation depth indicates that, in order to realize the interference modulation depth $M \geq$ 0.9 , the maximum value of the curve error of mirror $\varsigma$ should be $\varsigma_{\max } \leq \lambda / 8$. Figure 3 presents measured results of the surface of the plane mirror with the aid of Zygo interferometer. As can be seen from Figure 3, the plane mirror has a good surface, with the PV value $\lambda / 8$.

The interferometer is placed on the testbed, and the interference is formed by using the $\mathrm{He}-\mathrm{Ne}$ laser. The electrical signal of the laser interference can then be acquired. Figure 4 provides the interference signal generated by the laser light source.

As can be seen from Figure 4, the laser interference signal is a sinusoidal signal, and the amplitude of the interference signal is stable and the fringe is clearly visible, which meets the requirement of the interference modulation depth.

\section{Conclusions}

In the Fourier transform spectrometer, the Michelson interferometer is the core optical system. By focusing on the classic Michelson interferometer, we have conducted a theoretical analysis of the influence of surface shape of mirror and performance change of the beam splitter on the modulation depth. Theoretical deductions are obtained regarding tolerance requirements for the error of surface shape mirror and the reflection ratio of the beam splitter. The results obtained are applicable to the Fourier transform spectrometer within the framework of the Michelson dual beam interference.

\section{Conflict of Interests}

The author declares that there is no conflict of interests regarding the publication of this paper.

\section{Acknowledgment}

The research was supported by the Program for Science and Technology funded by the Education Department of Jiangxi Province (GJJ14316).

\section{References}

[1] B. Saggin, D. Scaccabarozzi, and M. Tarabini, "Instrumental phase-based method for Fourier transform spectrometer measurements processing," Applied Optics, vol. 50, no. 12, pp. 17171725, 2011.

[2] Y. Ferrec, J. Taboury, H. Sauer, and P. Chavel, "Optimal geometry for Sagnac and Michelson interferometers used as spectral imagers," Optical Engineering, vol. 45, no. 11, Article ID 115601, 2006.

[3] B. J. Meers and K. A. Strain, "Modulation, signal, and quantum noise in interferometers," Physical Review A, vol. 44, no. 7, pp. 4693-4703, 1991.

[4] H. Liang and P.-G. Wang, "Effects of low temperature on modulation efficiency of michelson interferometer," Acta Photonica Sinica, vol. 38, no. 4, pp. 967-970, 2009.

[5] Z.-C. Bu, C.-M. Zhang, B.-C. Zhao, and H.-C. Zhu, "Analysis and calculation of the modulation depth of the Michelson interferometer with wide field, chromatic compensation and thermal compensation," Acta Physica Sinica, vol. 58, no. 4, pp. 2415-2422, 2009.

[6] P. K. Katti and K. Singh, "A note on the surface accuracy and alignment of the end mirrors in a michelson interferometer," Applied Optics, vol. 5, no. 12, pp. 1962-1964, 1966.

[7] L. Shi, K. Li, Z.-F. Gao, L.-B. Zeng, and Q.-S. Wu, "Design of a compact structure interferometer," Spectroscopy and Spectral Analysis, vol. 33, no. 8, pp. 2294-2298, 2013.

[8] D. Y. Yu and H. Y. Tan, Engineering Optics, Mechanical Industry Press, Beijing, China, 2006. 

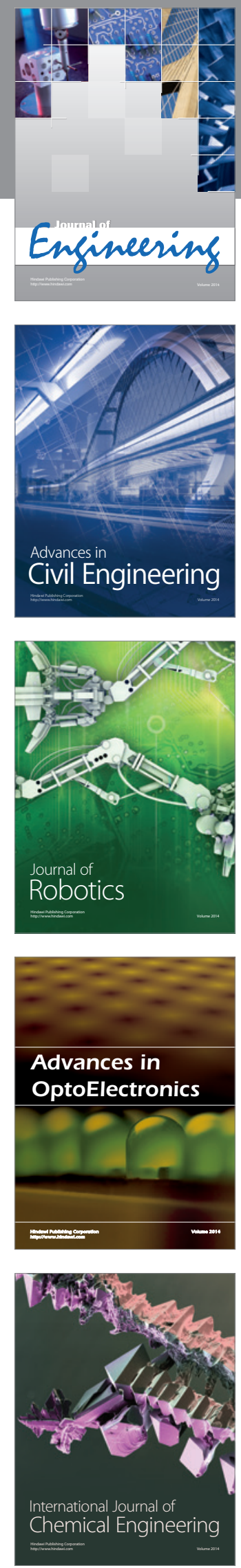

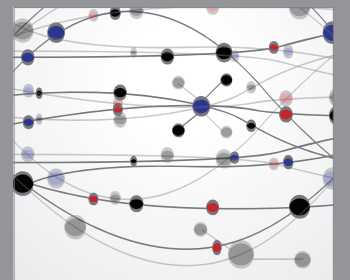

The Scientific World Journal
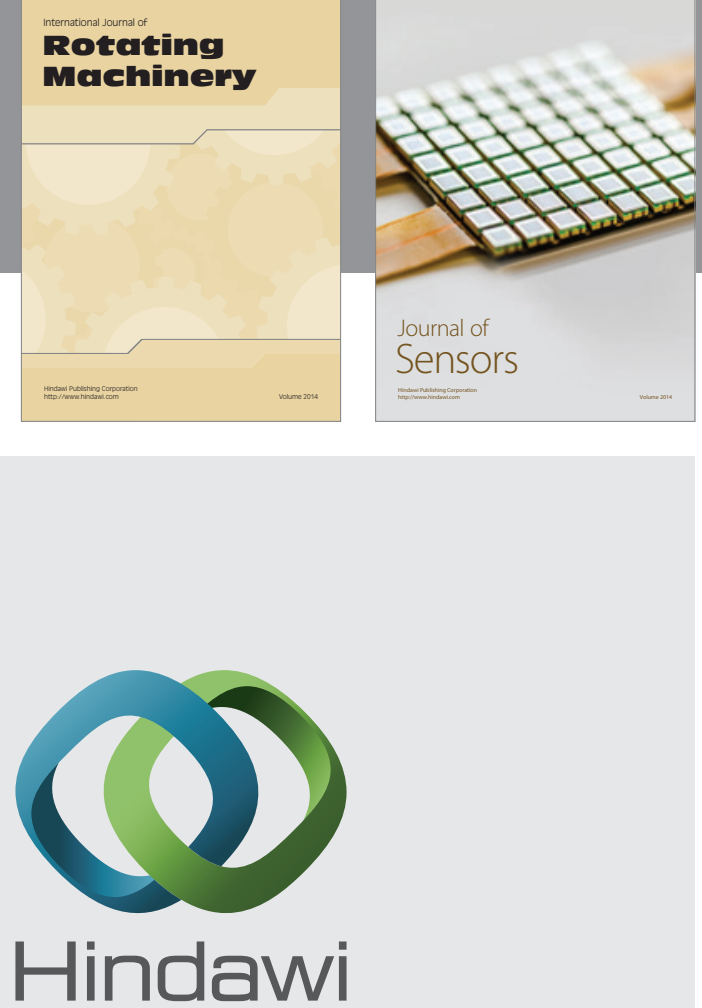

Submit your manuscripts at http://www.hindawi.com
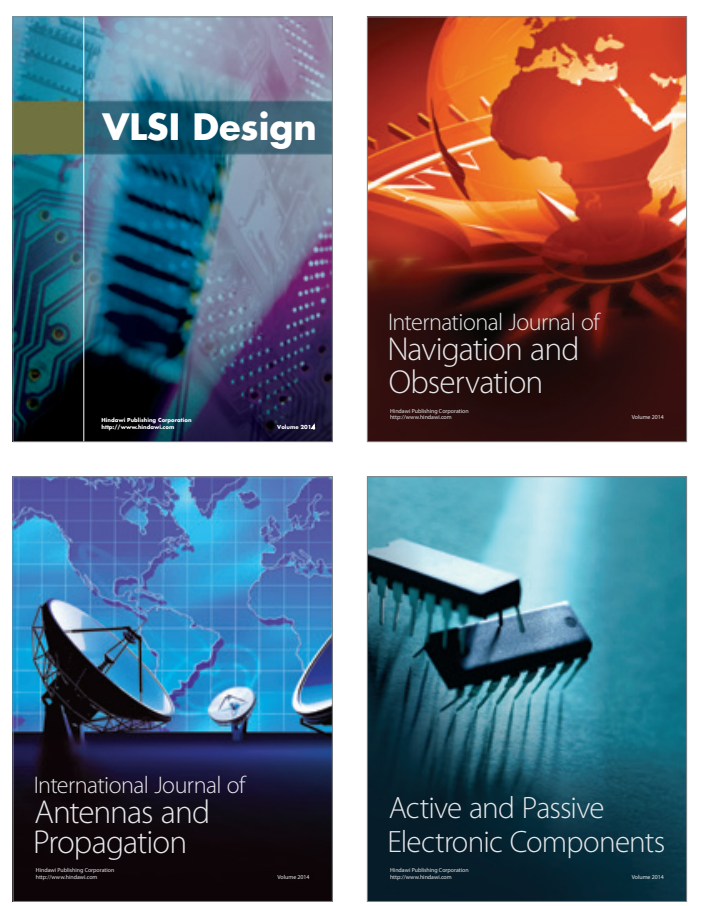
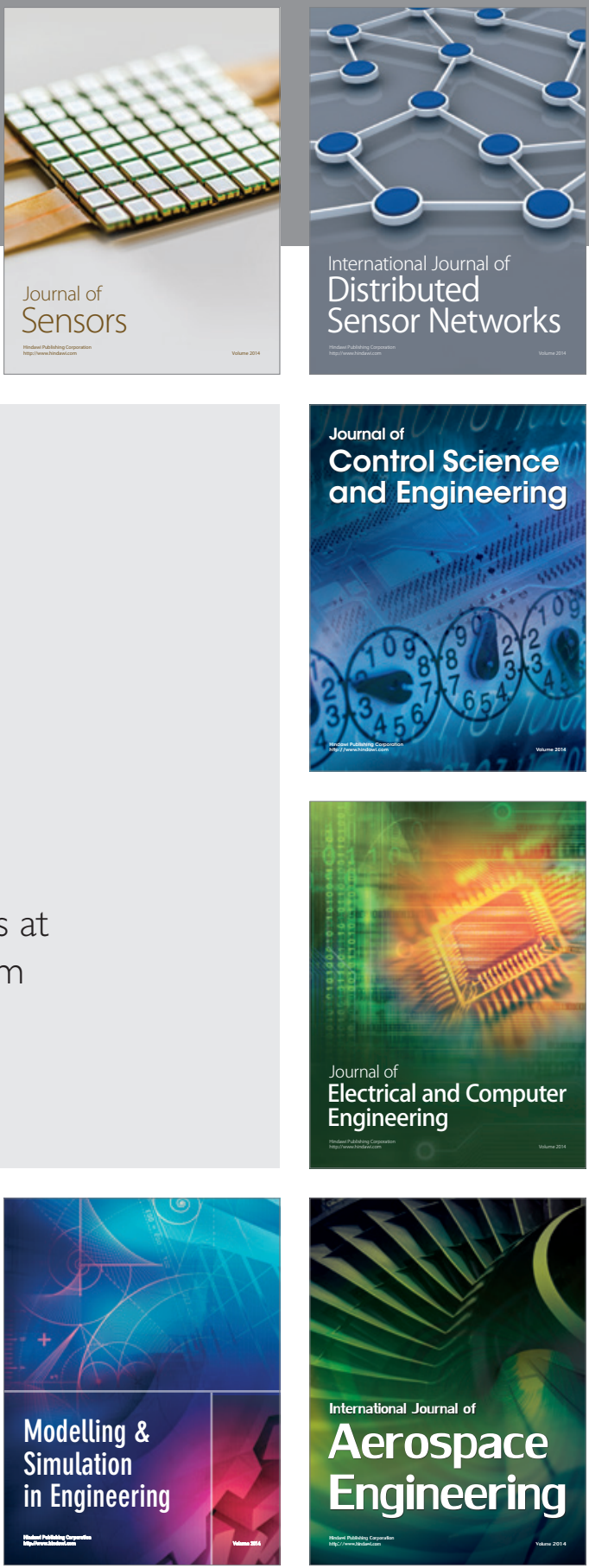

Journal of

Control Science

and Engineering
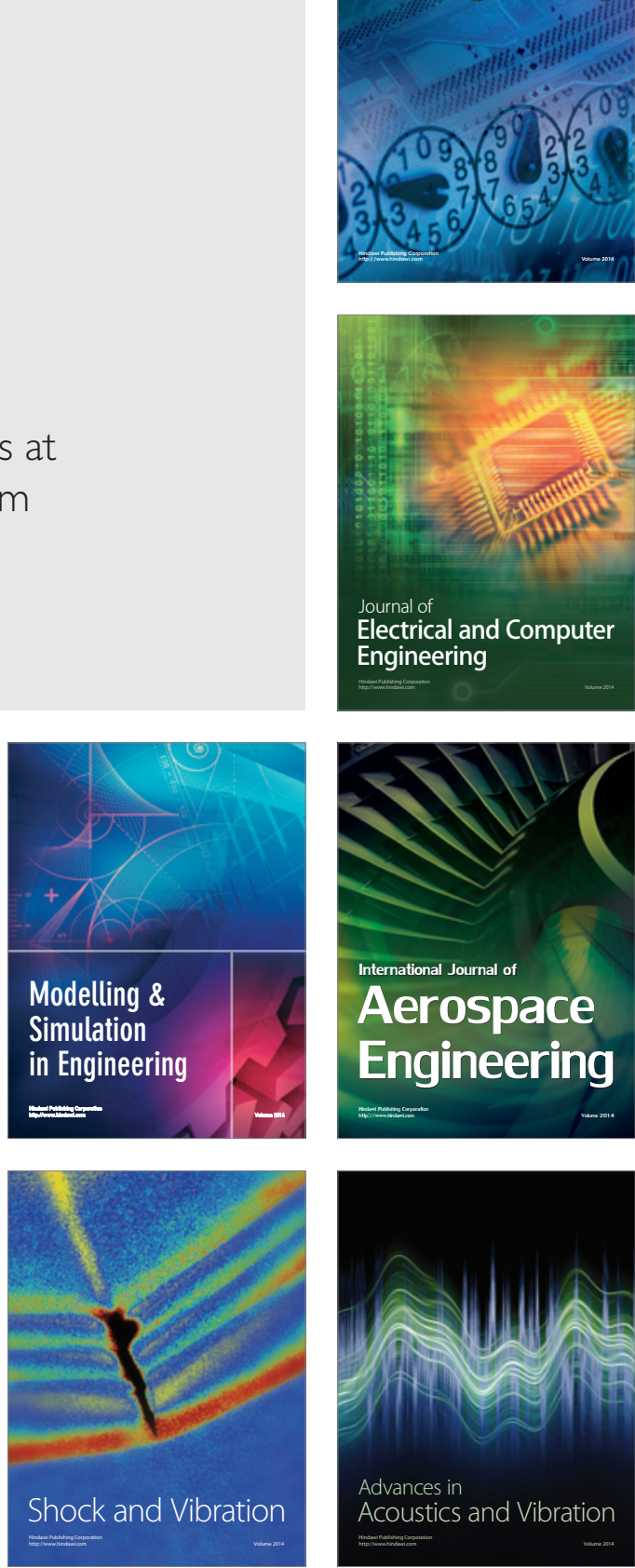